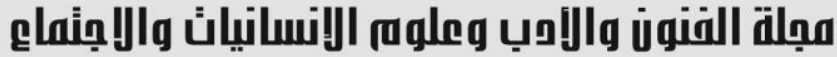

Journal of Arts, Literature, Humanities and Social Sciences www.jalhss.com

\section{الفروق الجندرية في الصحة العقلية}

مركز دراسات المر أة مهابي - جامعة بغداد ـ العراق reem.m@wsc.uobaghdad.edu.iq الايميل

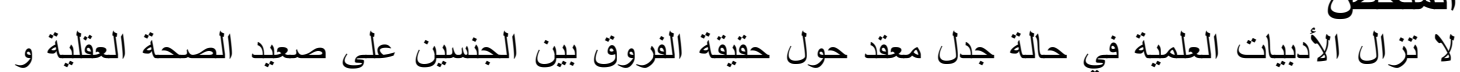

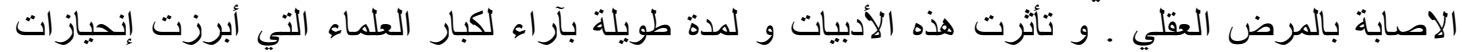

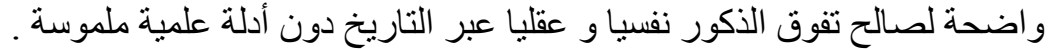

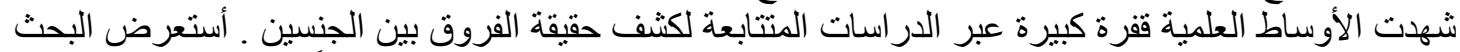

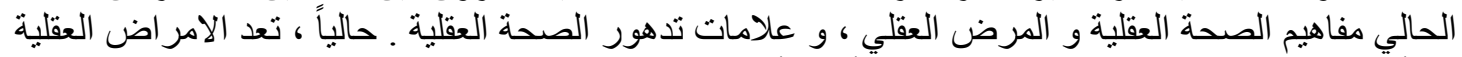

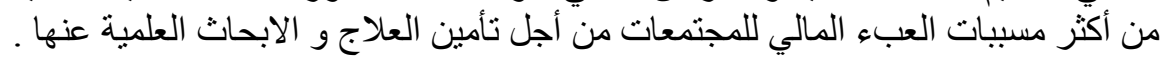

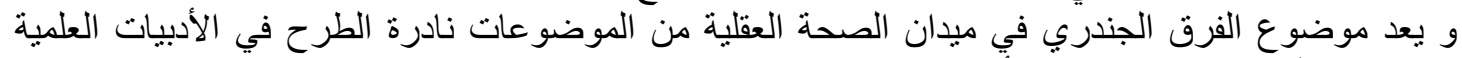

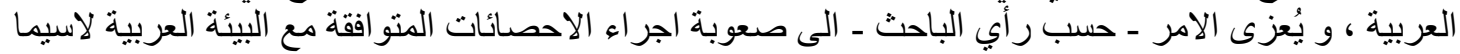

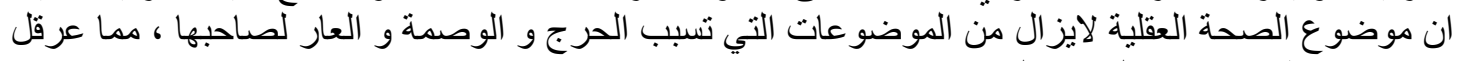

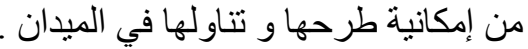

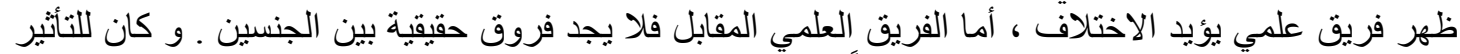

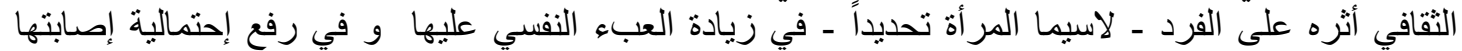

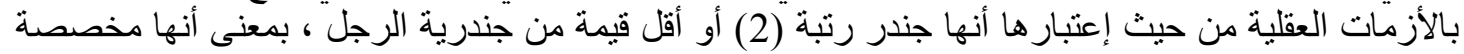

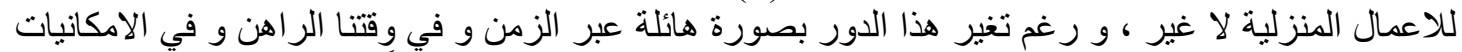

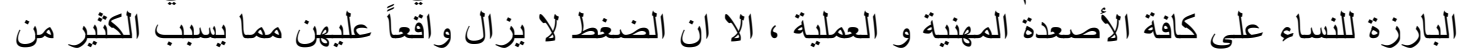

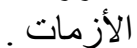

في الخاتمة ، هناك فروق جندرية فيما يتعلق بنوعية الاضطر ابابات العقلية ، لكن يتطلب ايجاد الفروق الجنات الجندرية

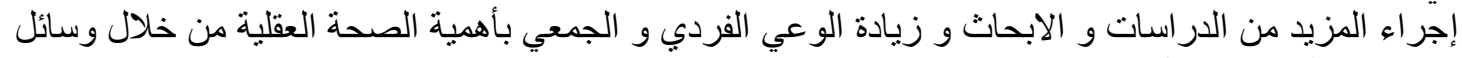

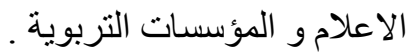




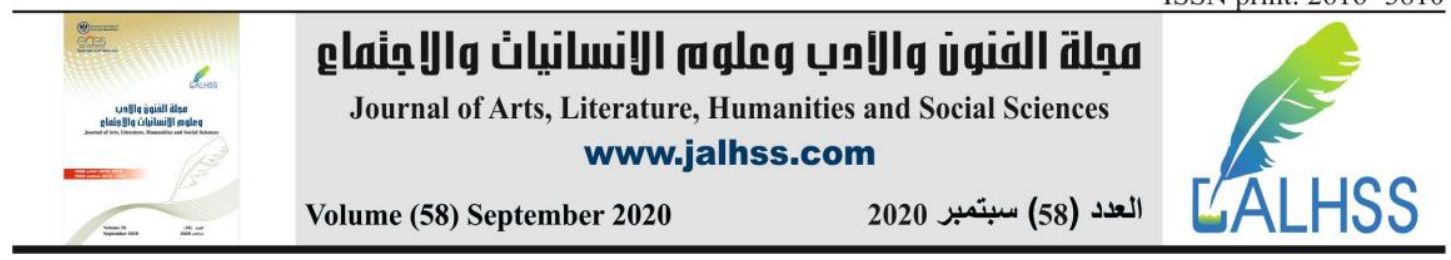

\title{
Gender Differences in Mental Health
}

\author{
Dr. Reem Kh. Mahdi \\ Women's Studies Center - University of Baghdad - Iraq \\ Email: reem.m@wsc.uobaghdad.edu.iq
}

\begin{abstract}
The scientific literature is still in a state of complex controversy about truth of the Gender differences in terms of mental health and mental illness. This literatures were influenced for a long time by views of senior scholars that highlight clear biases in favor of male superiority psychologically and mentally throughout history without tangible scientific evidences.

Scientific community witnessed a large leap through successive studies to reveal reality of gender differences. The current research showed concepts of mental health and mental illness, and signs of mental health impairment. Currently, mental illnesses are among the most common causes of financial burden in the fields in order to secure treatment and scientific research on them .

The gender difference in mental health is one of the rare topics raised in the Arab scientific literatures, and the matter is attributed to the difficulty of conducting statistics compatible with the Arab environment, especially in the issue of mental health is still among the topics That causes embarrassment, stigma and shame for its owner, which hindered the possibility of being put up and taken up in the field.

There is a scientific team that supports the difference, while the other doesn't find real differences.

Cultural impact had its role also on the individual - especially on the woman - by increasing the psychological burden on her and in raising the possibility of developing mental illness in terms of considering that she is a gender (2) or less valuable than men, in the sense that it's devoted to domestic work only, and despite change This role tremendously over time and in the present time and in prominent capabilities of women at all levels, professional and practical, but the pressure is still on them, which causes many crises.

Conclusion: there are gender differences in quality of mental disorders, but finding gender differences requires further studies and increasing individual and collective awareness of mental health through media and educational institutions .
\end{abstract}

Keywords: Gender, Differences, Mental Health. 


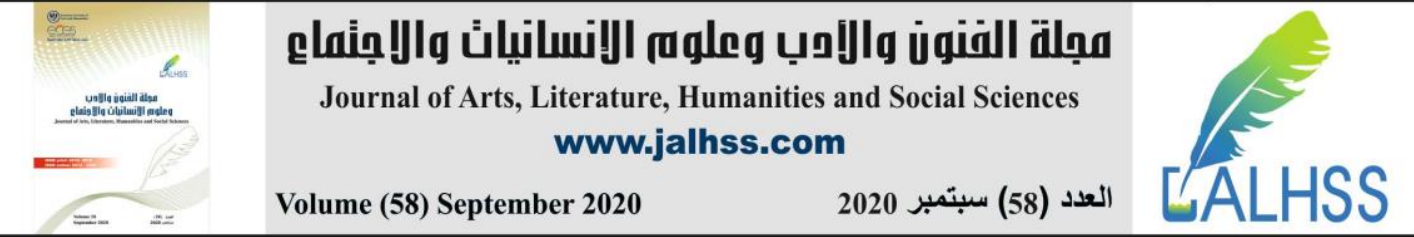

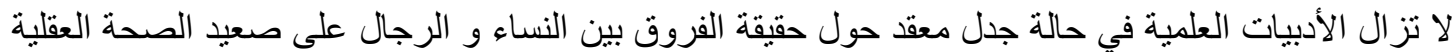

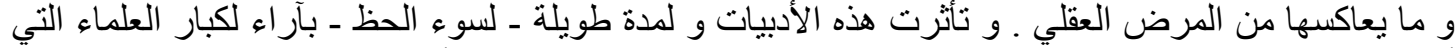

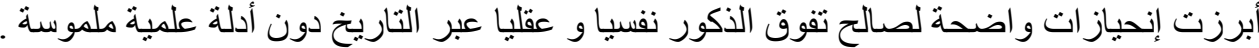

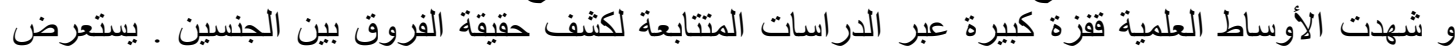

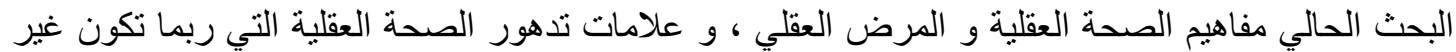

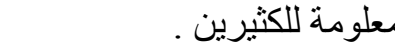

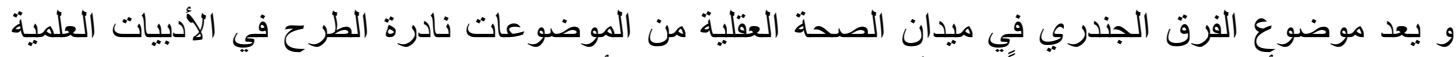

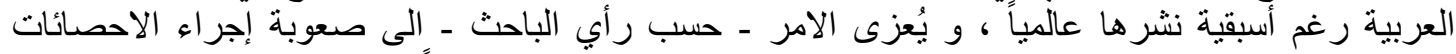

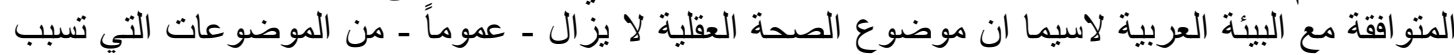

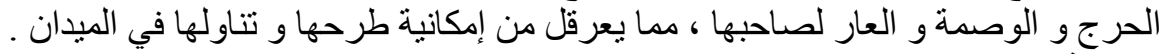

مشكلة البحث : مالبهة

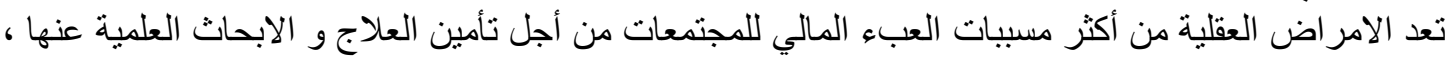

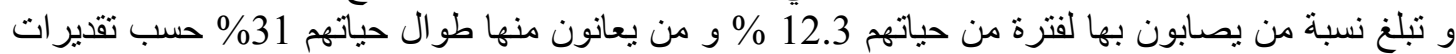

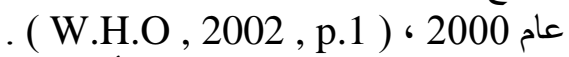

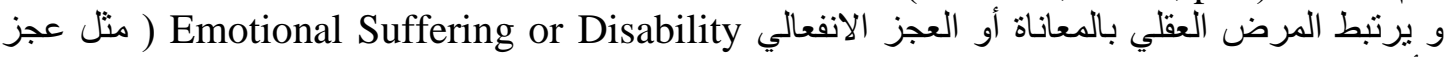

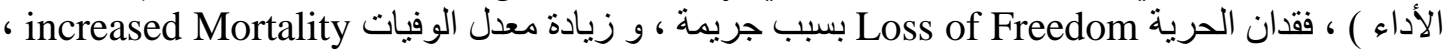

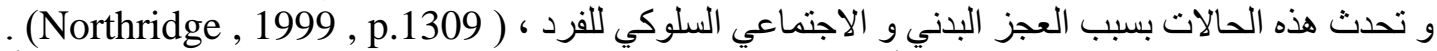

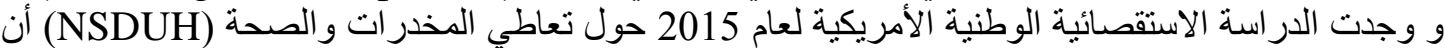

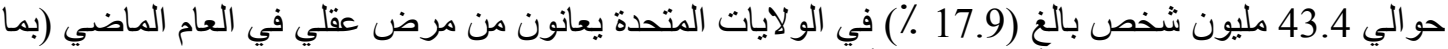

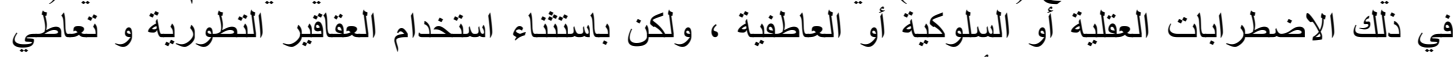

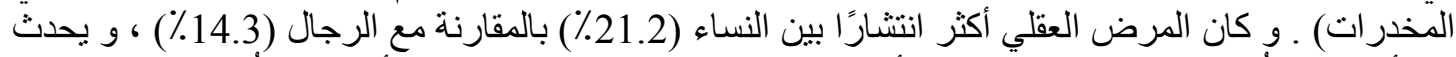

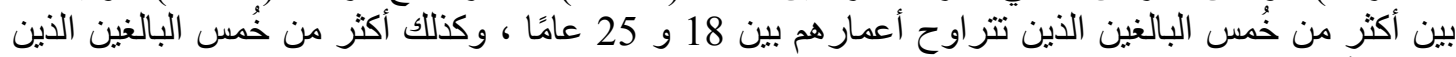

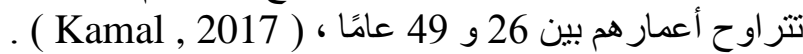

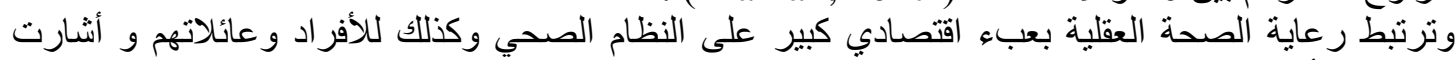

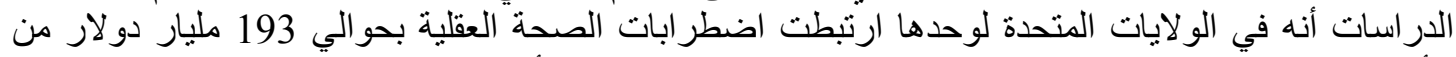

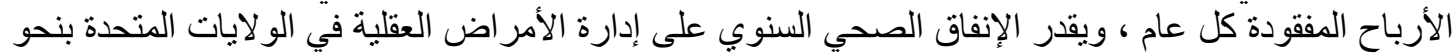

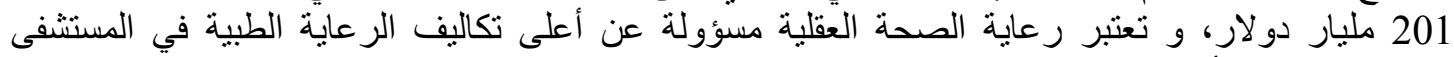

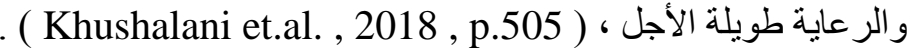

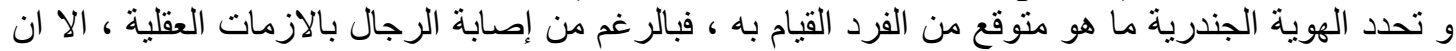

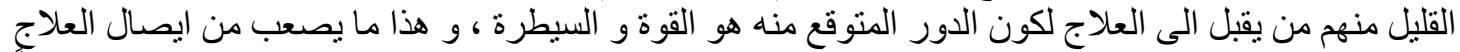

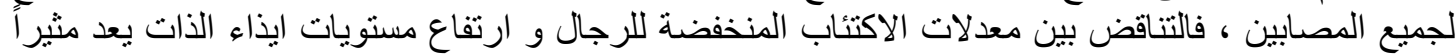

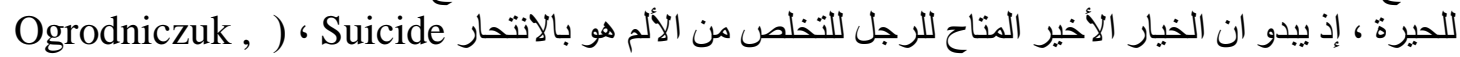

. (Oliffe, Kuhl \& Gross , 2016

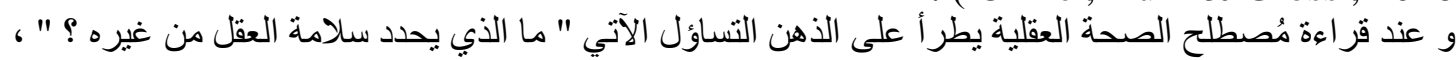

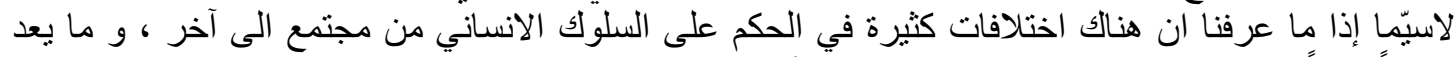

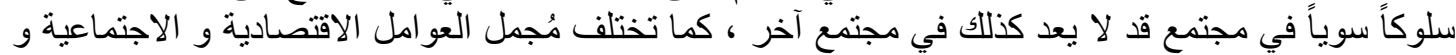

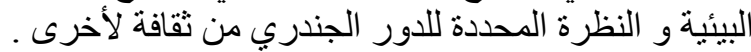

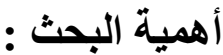

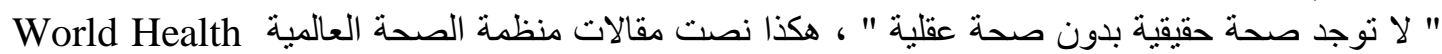
و Organization

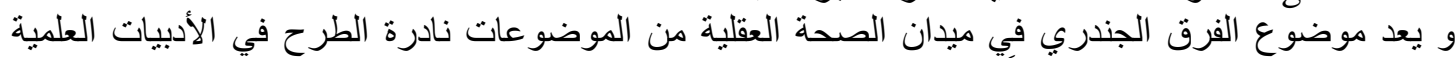

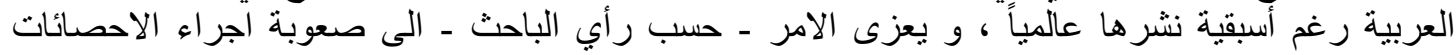




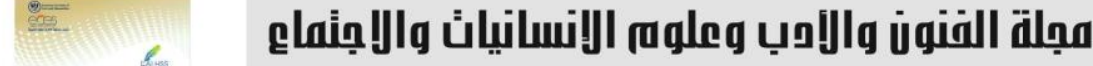 \\ Journal of Arts, Literature, Humanities and Social Sciences www.jalhss.com

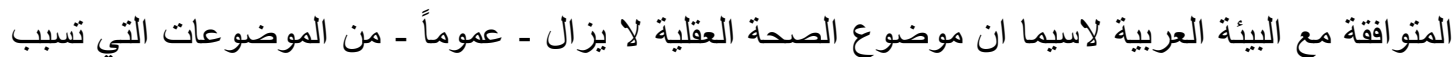

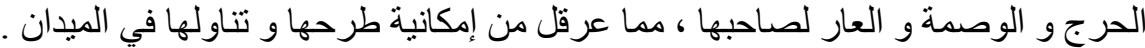

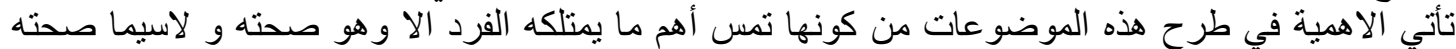

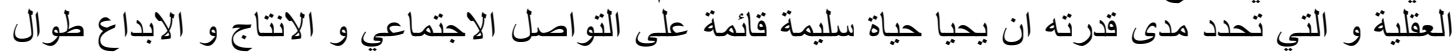

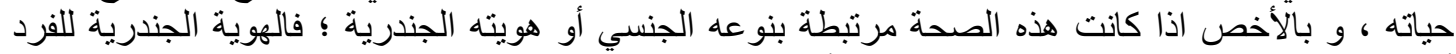

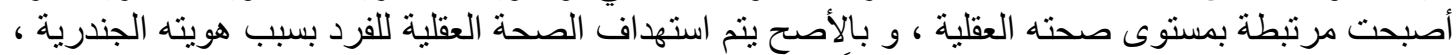

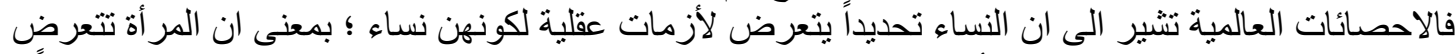

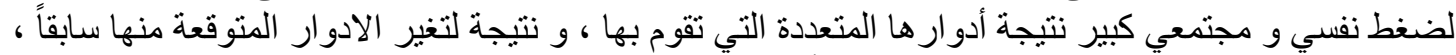

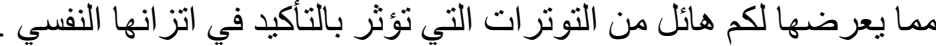

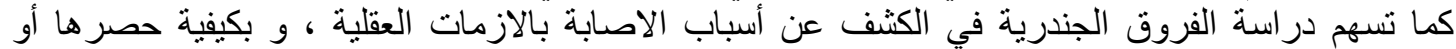
علاجها بطرق أفضل بطرق هرمونية أو نفسية تناسب كل جندر على حده ، ( Riecher-Rossler , 2018 ) .

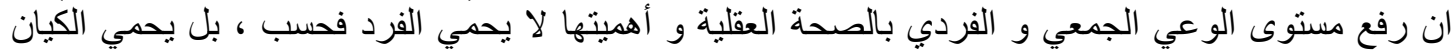

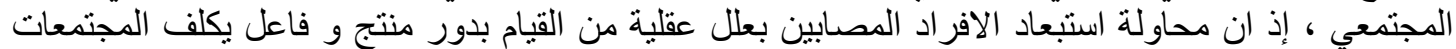

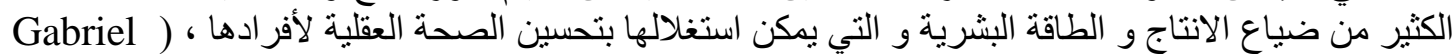

. ( \& Liimatainen , 2000

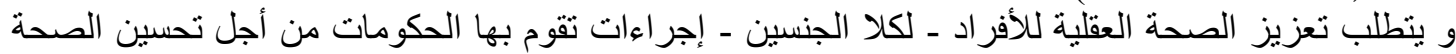

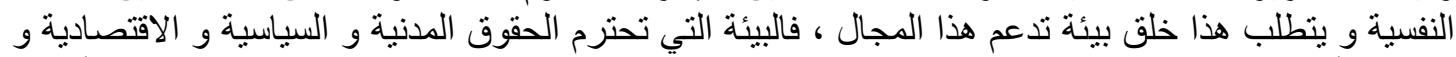

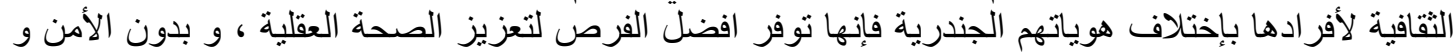

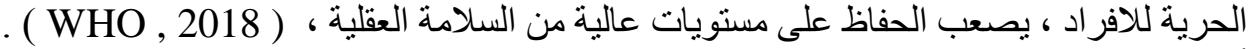

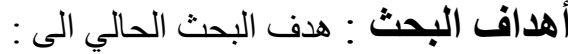

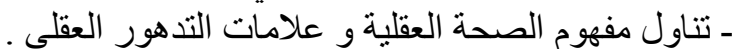

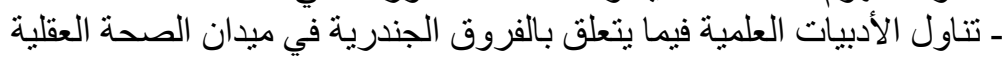

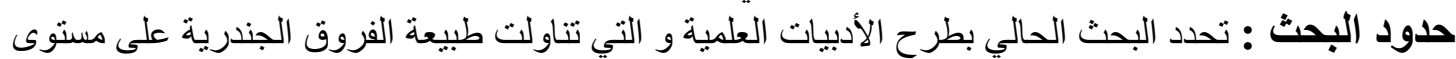
الصحة العقلية.

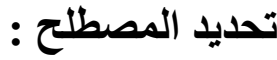

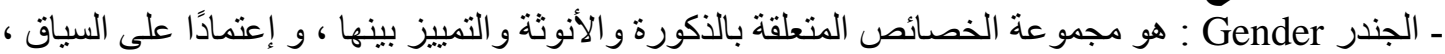

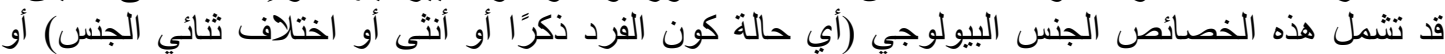

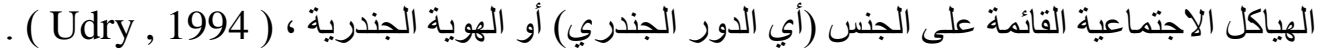

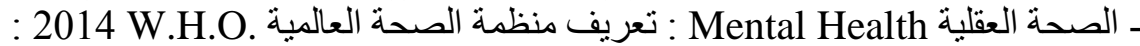

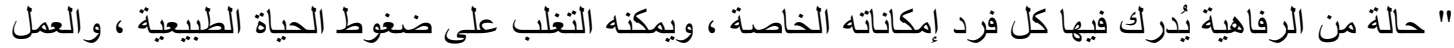

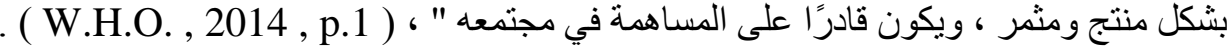

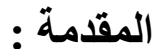

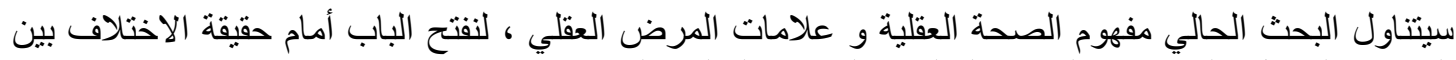

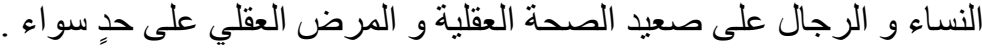

تاريخ استعمال مُصطلح الصحة العقلية :

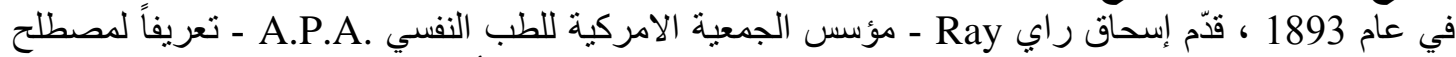

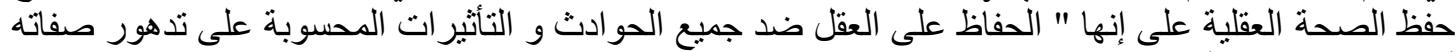
( Mandell , 1997 , p.1 )

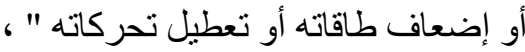

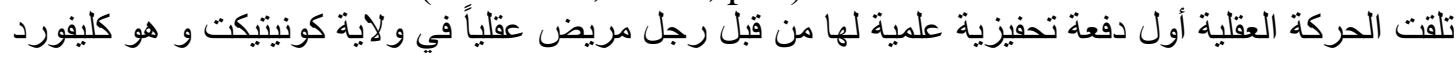

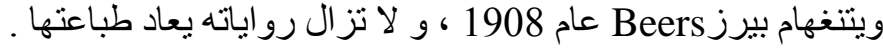




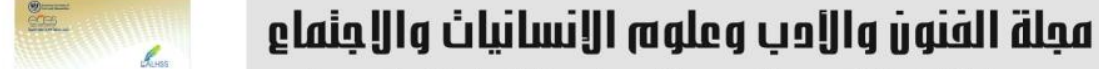 \\ Journal of Arts, Literature, Humanities and Social Sciences www.jalhss.com

" عقل وجد نفسه " أصبحت رواية ملهمة للطلبة و الباحثين المختصين بالصحة العقلية ـو و بمساعي منه و من

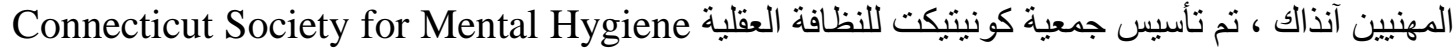
. (Britannica , 2014 ) ،

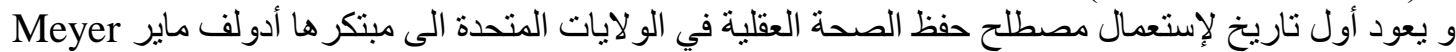

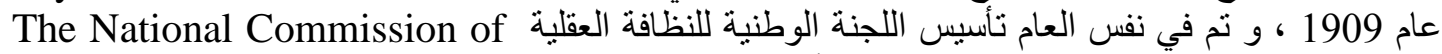
Mental Hygiene أفريقياو هنغاريا.

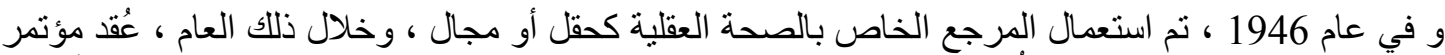

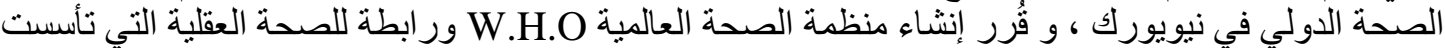

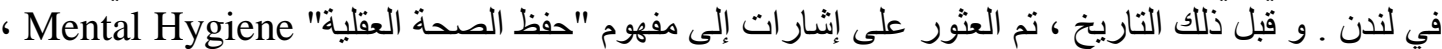

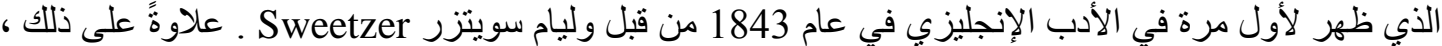

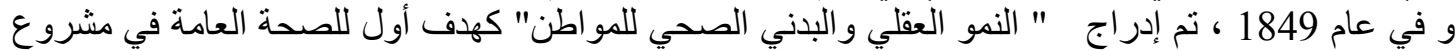

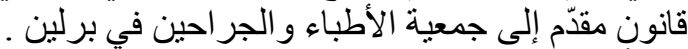

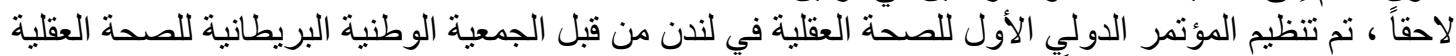

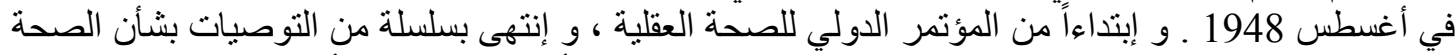

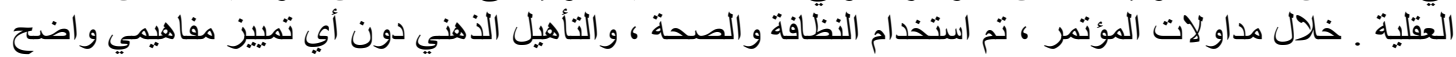

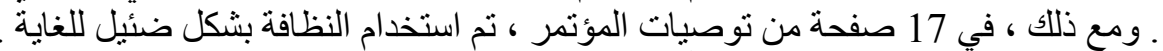

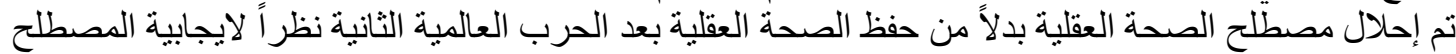

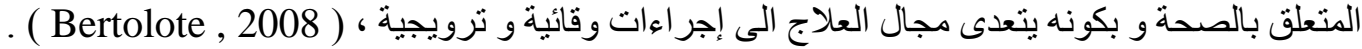
مظاهر الصحة العقلية السليمة :

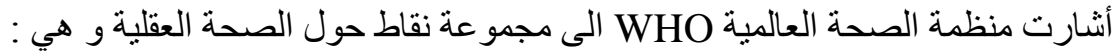

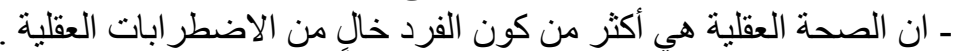

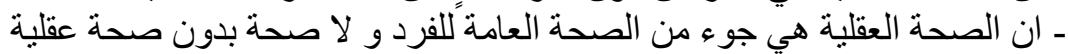

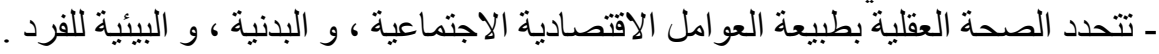

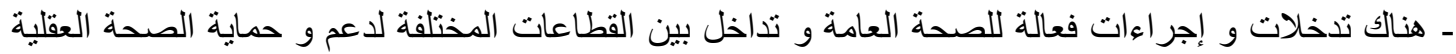

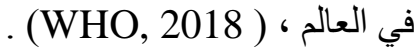
علامات تلهور الصحة العقلية : عالإلية

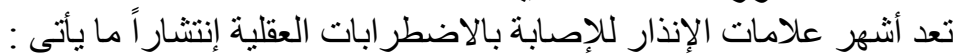

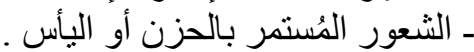

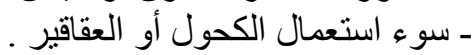

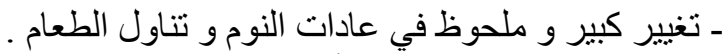

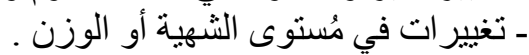

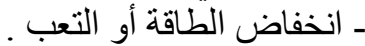

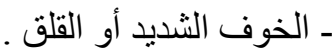

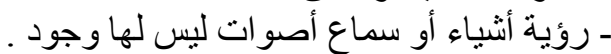

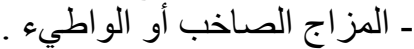
ـ ـ الأوجاع أو الصداع أو مشأكل في الجهاز الهضدي دون سبب و اضح.

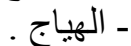

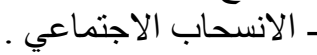
ـ التفكير في الانتحار ، ( NIMH , 2019 ) .

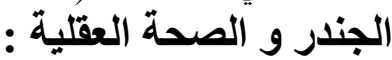

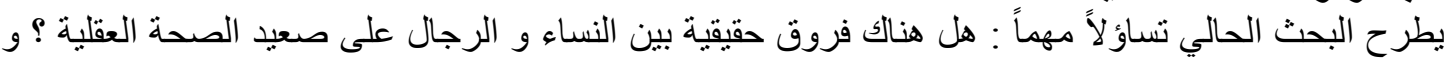

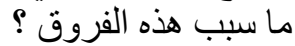




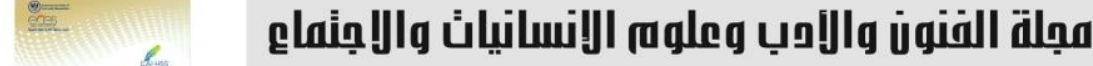 \\ Journal of Arts, Literature, Humanities and Social Sciences www.jalhss.com \\ Volume (58) September 2020

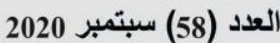

تثير الأدلة العلمية الحالية الى عدم وجود فروق جندرية على صعيد نسب الاصـابة بالاضطر ابات العقلية ، لكنها

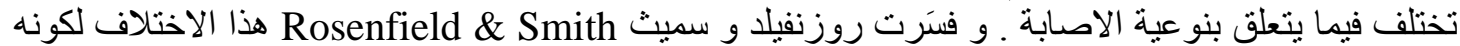

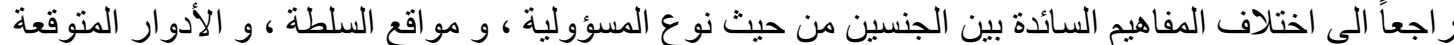

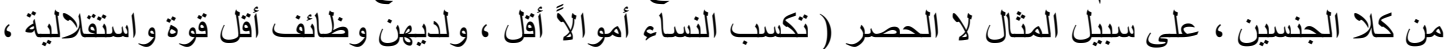

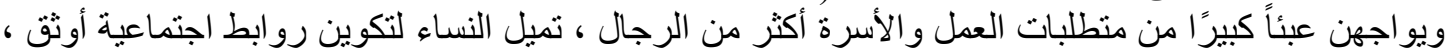

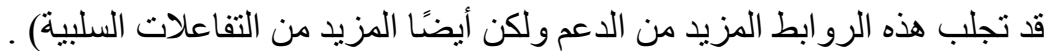

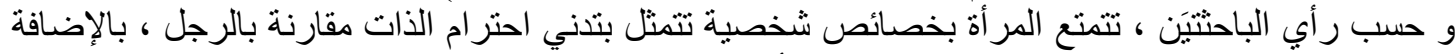

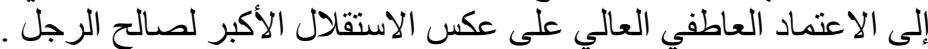

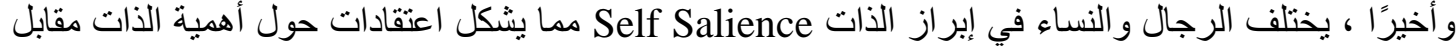

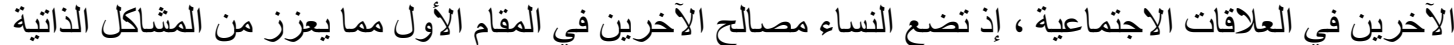

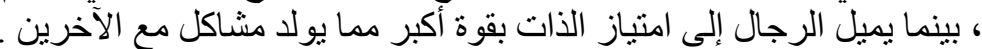

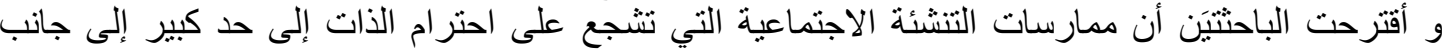
احتر ام الآخرين للآخرين تنتمَي الصحة العقلية للفرد ، ( Rosenfield \& Smith , 2012 ) .

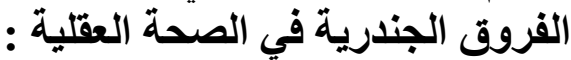

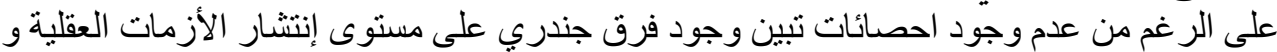

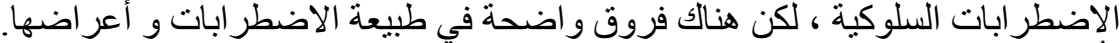

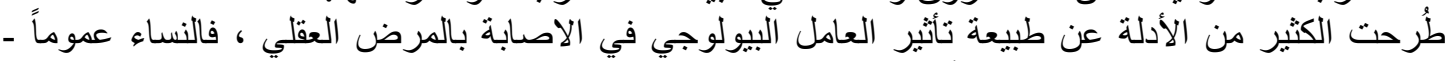

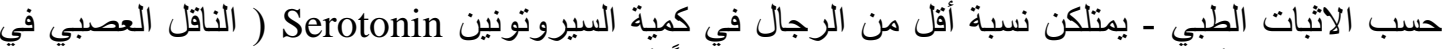

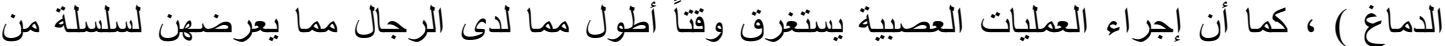

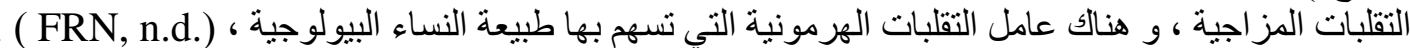

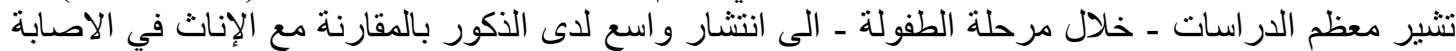

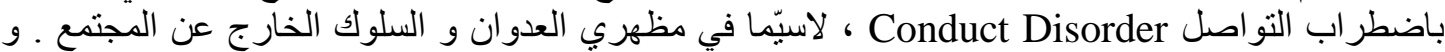

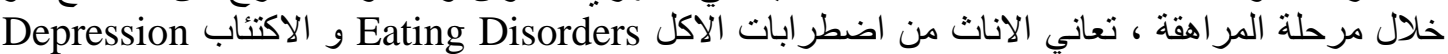

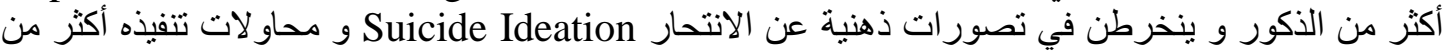

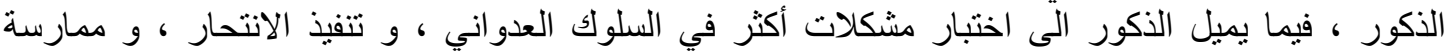

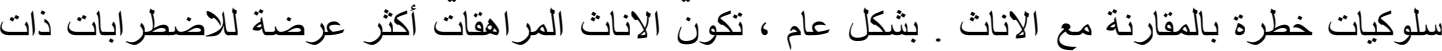

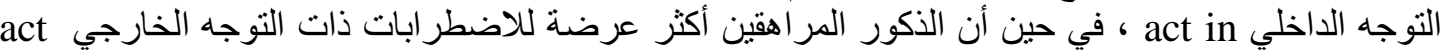

out

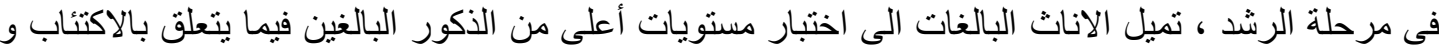

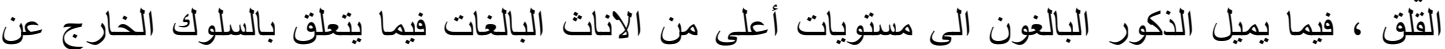

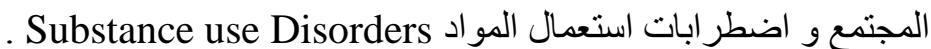

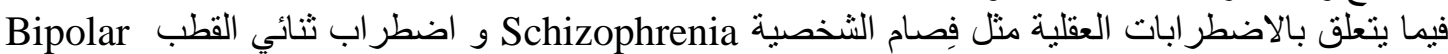

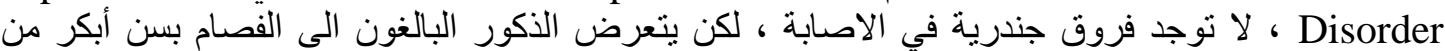

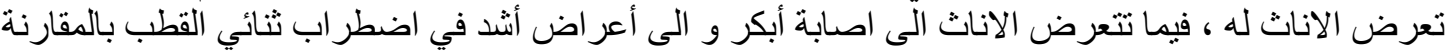

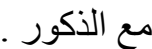

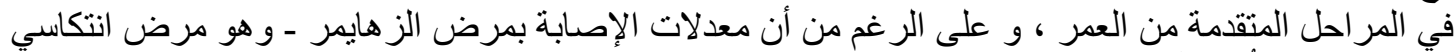

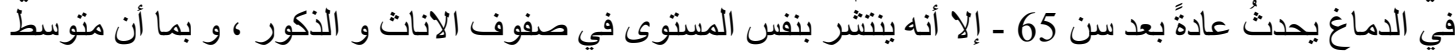

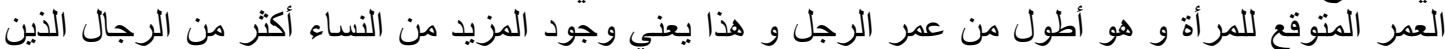

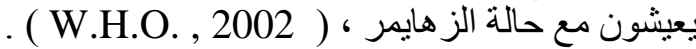

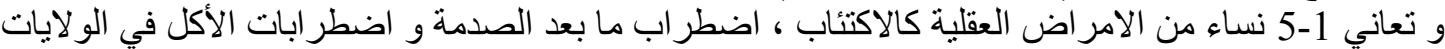

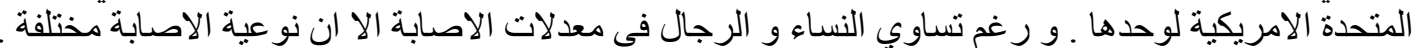

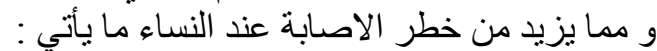

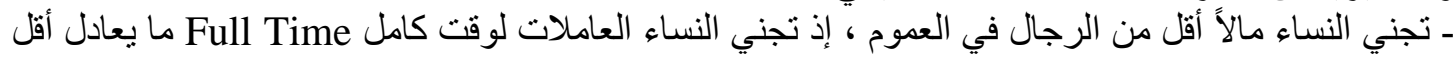

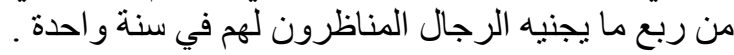




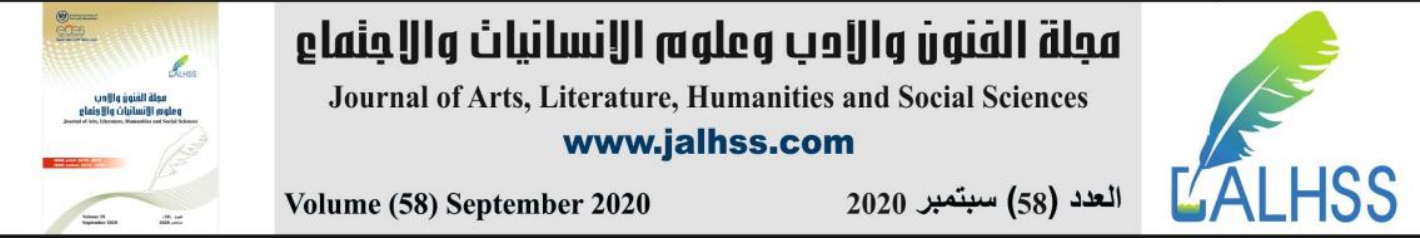

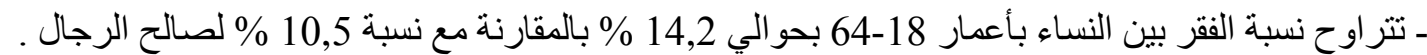

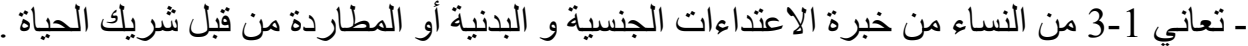

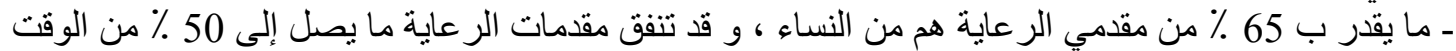

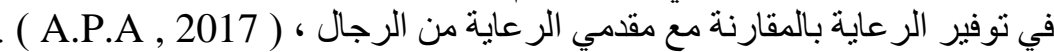

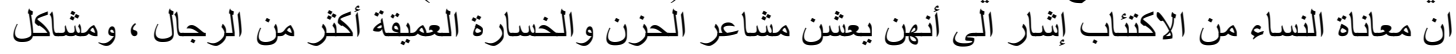

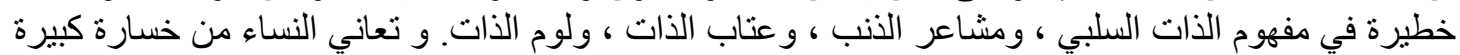

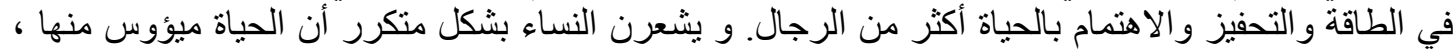

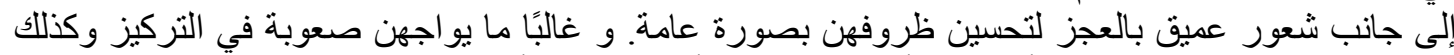

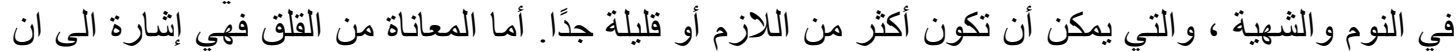

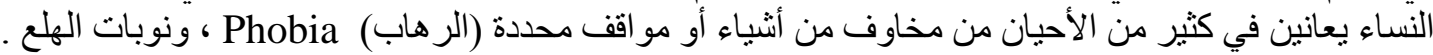

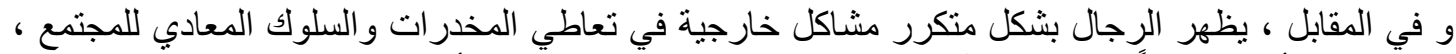

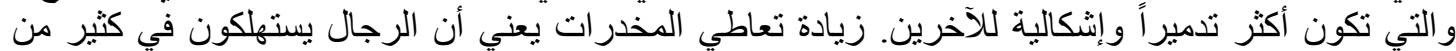

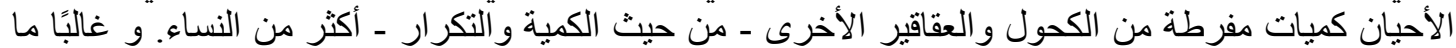

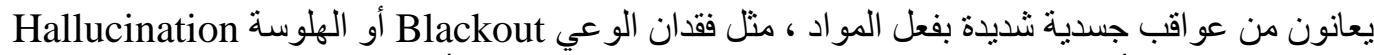

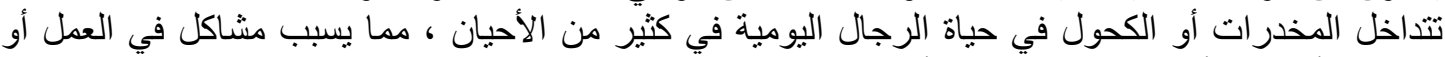

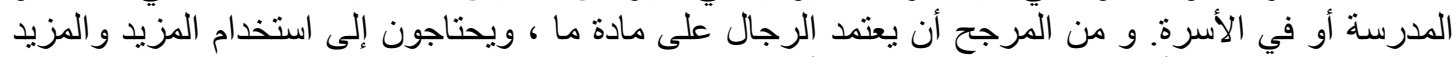

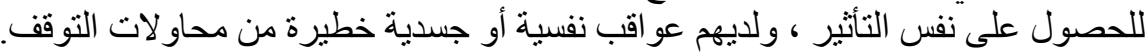

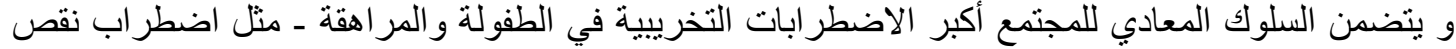

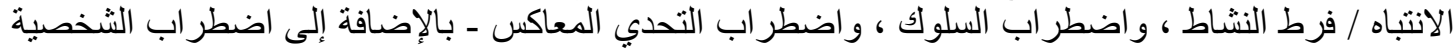

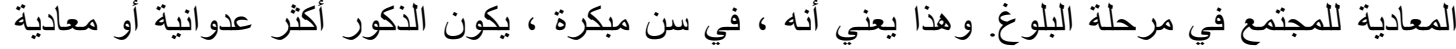

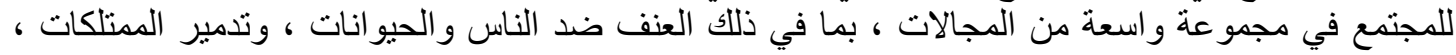

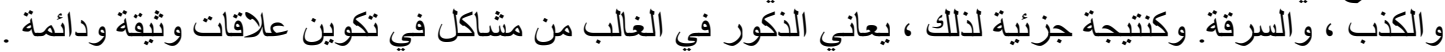

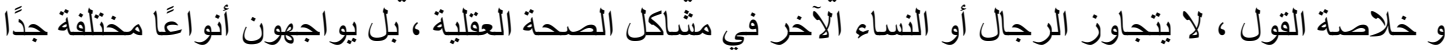

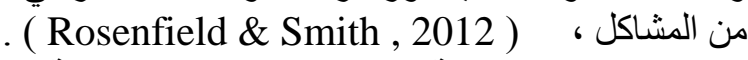

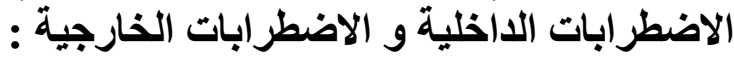

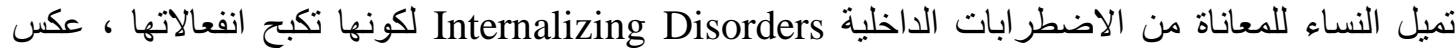

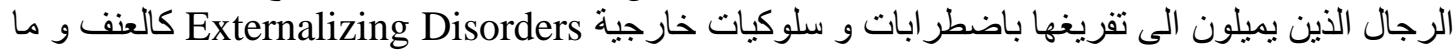

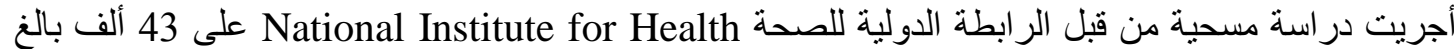
امريكي من كلا الجنسين عام 2001 ، و نم تحديد المتغير ات الديمو غرية الفية ذات الصلة ( العمر ، العرق ، الجندر

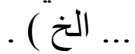
بعد إجراء المقاييس التشخيصية للاضطر ابات ، كانت نسبة 22,9\% من الاصنابة بالاكتئاب ( اضطر اب داخلي )

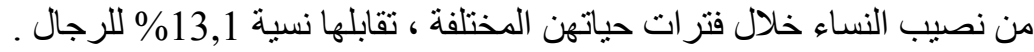

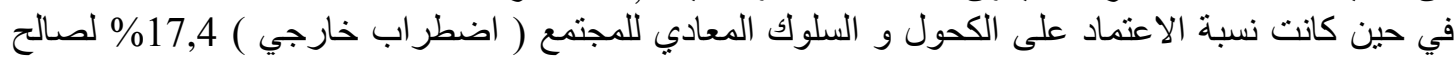

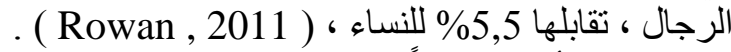

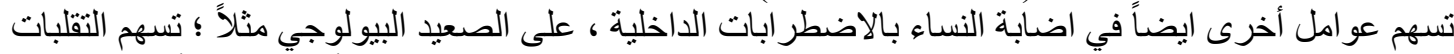

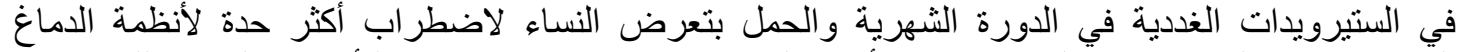

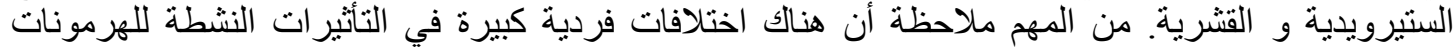

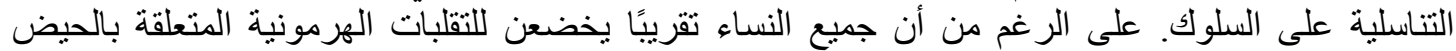

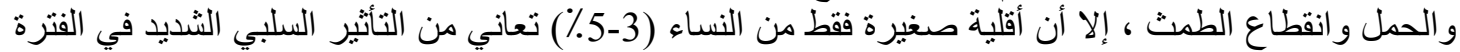

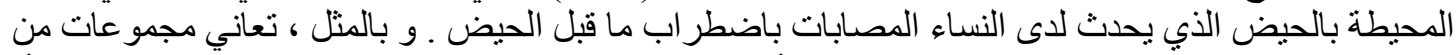

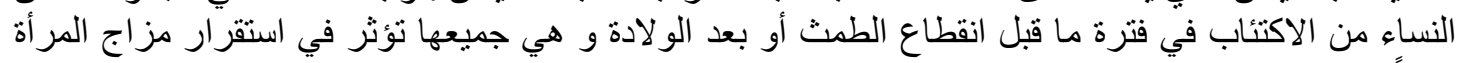

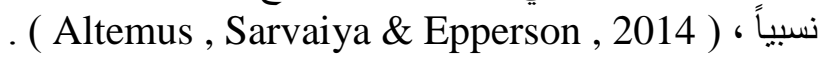




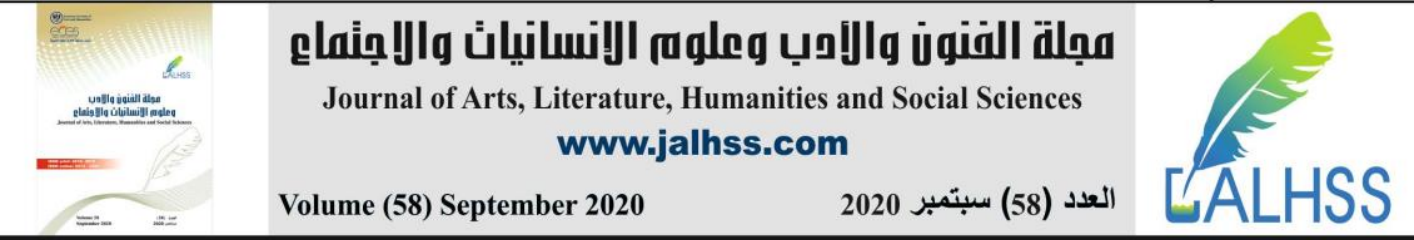

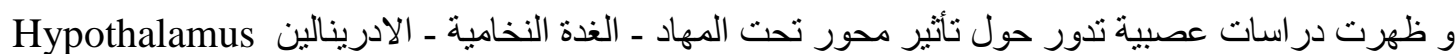
مPA Axis- - Pituitary - Adrenal في طريقة الاستجابة للضغوط

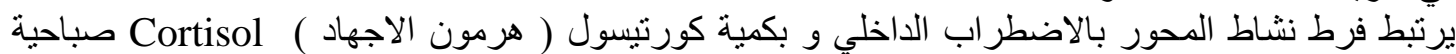

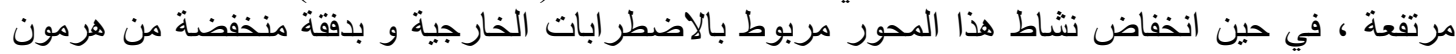

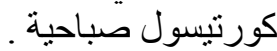

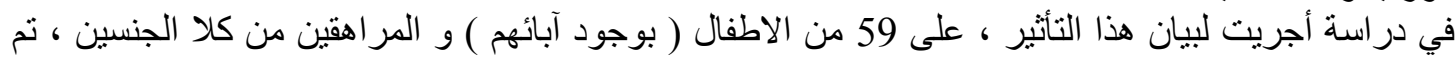

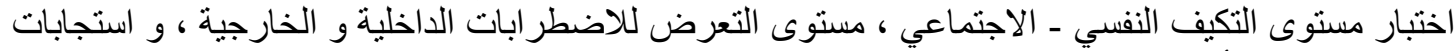
المحور HPA للأداء و الضغوط النيف النفسية .

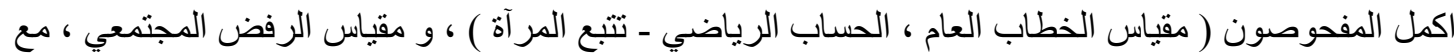

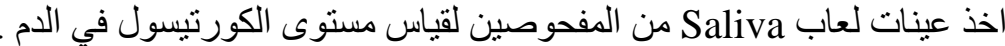

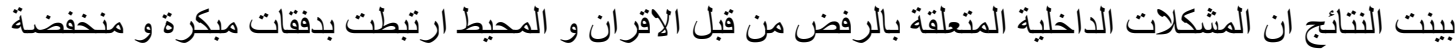

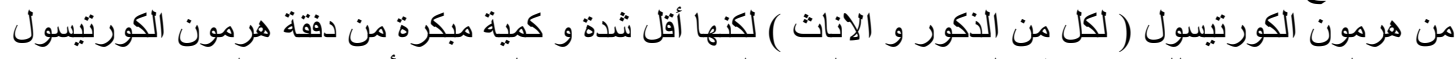

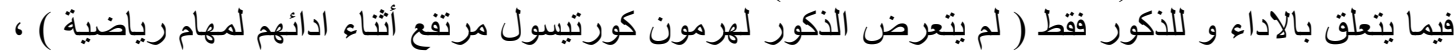

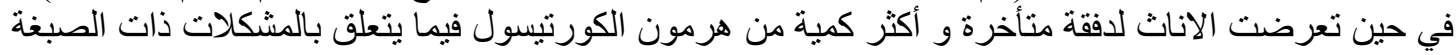

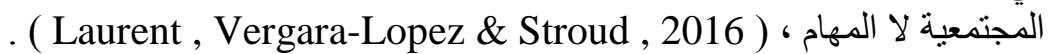

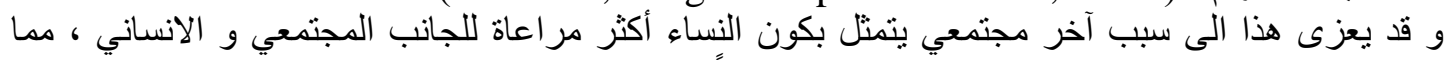

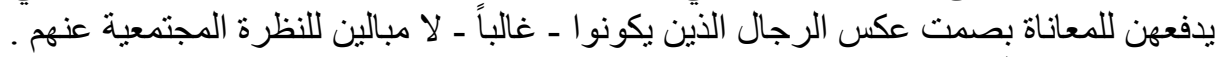

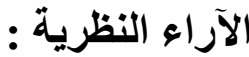

التحليل النفسي Psychoanalysis : الأفرو : فروق جندرية لصالح الذكر

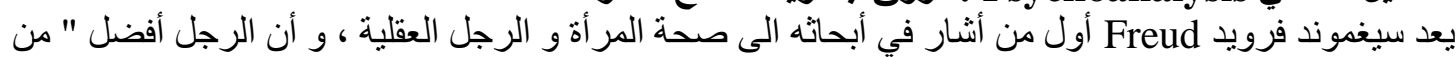

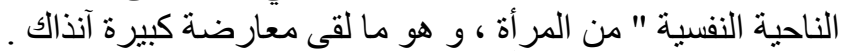

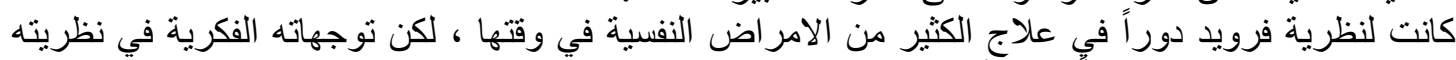

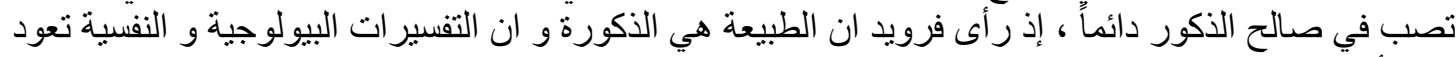

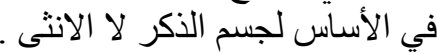

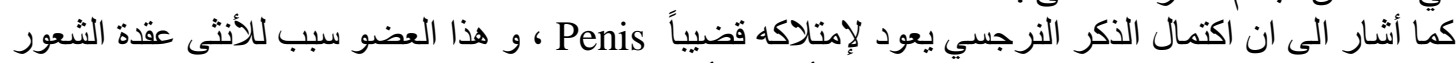

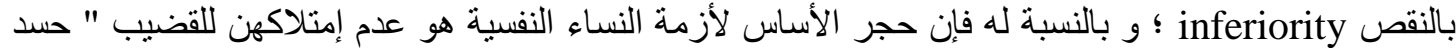

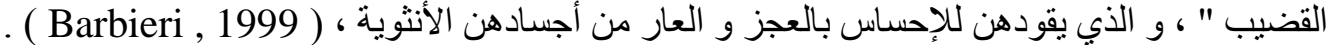

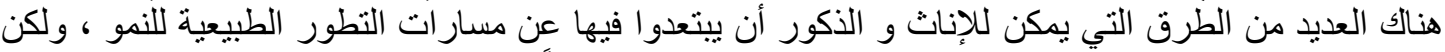

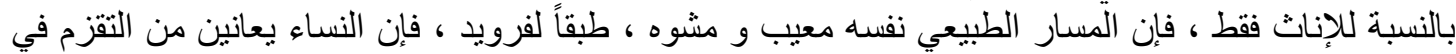

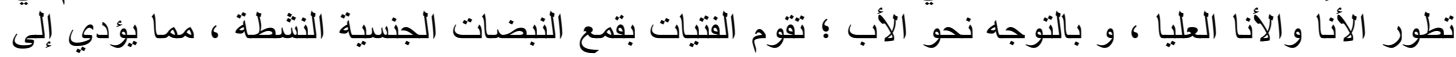

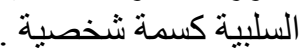

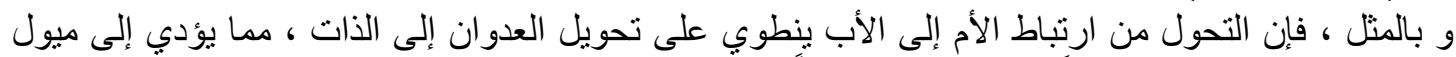

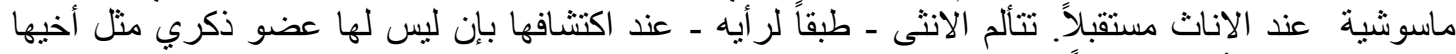

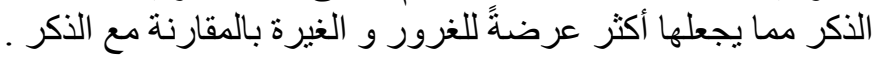

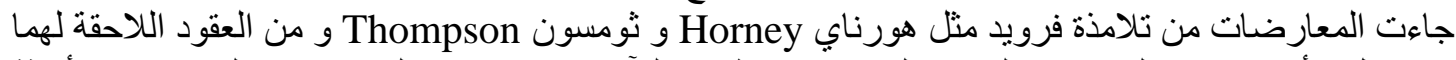

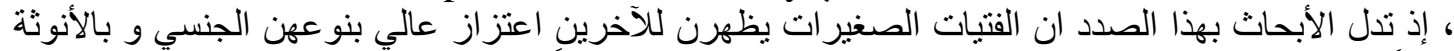

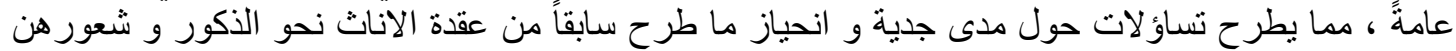

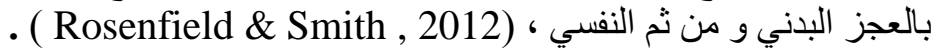

هايد Janet Shibley Hyde : لا فروق جندرية كبيرة

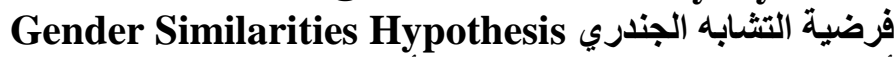

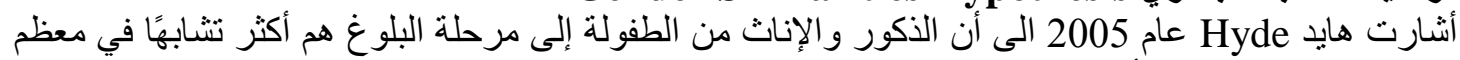

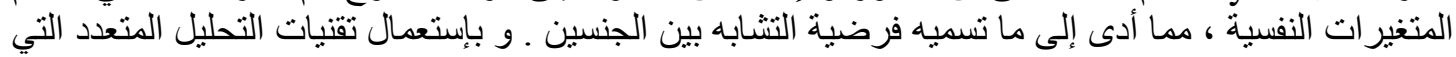




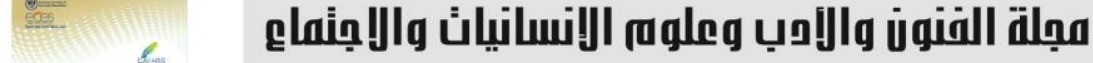 \\ Journal of Arts, Literature, Humanities and Social Sciences www.jalhss.com

أحدثت ثورة في دراسة الاختلافات بين الجنسين بدءًا من الثمانينيات ، حلت كيف أجرت الأبحاث السابقة تقييم

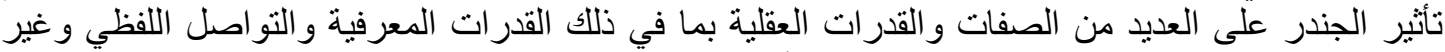

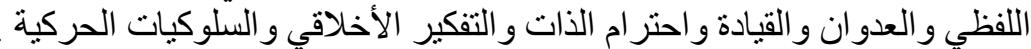

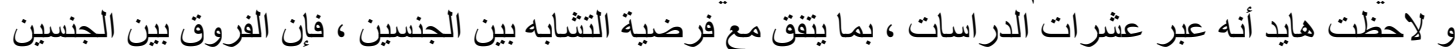

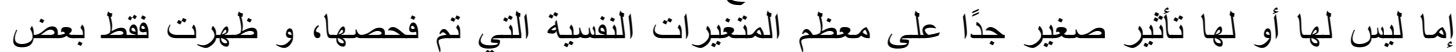

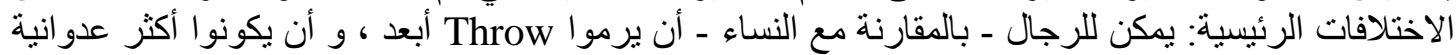

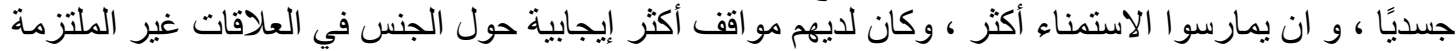

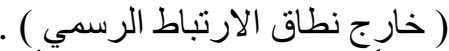

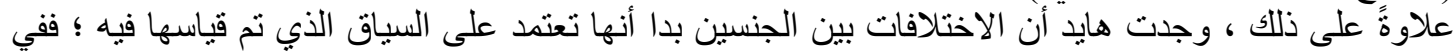

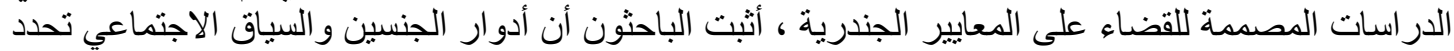

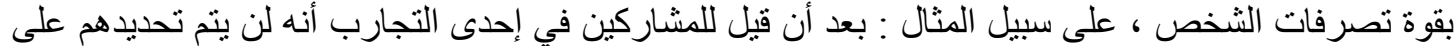

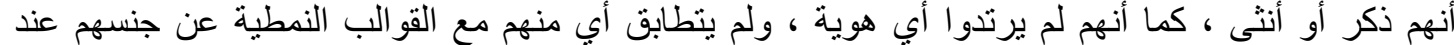

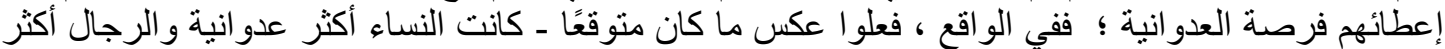

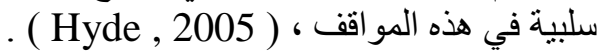

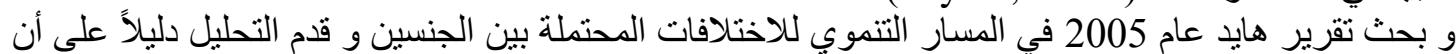

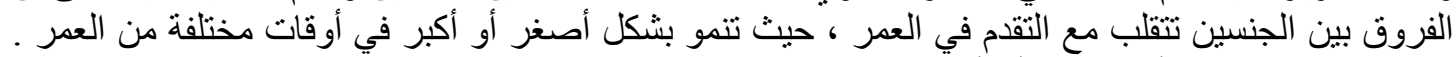

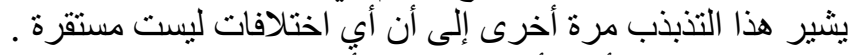

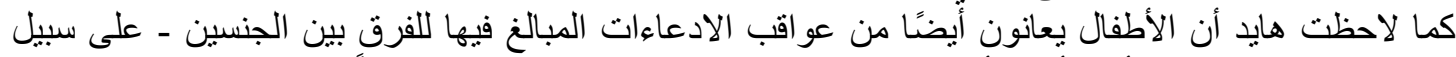

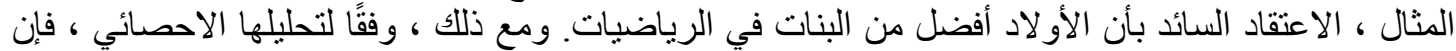

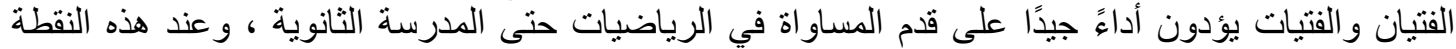

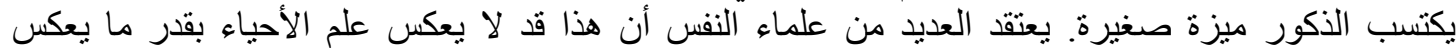

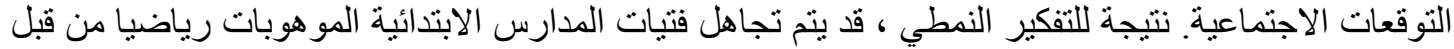

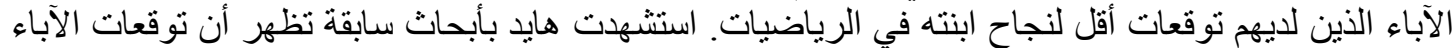

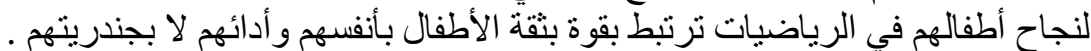

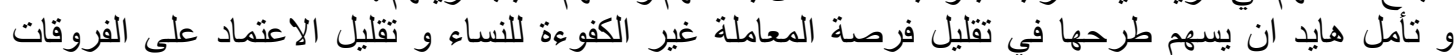

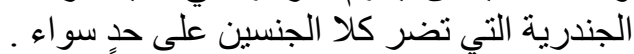

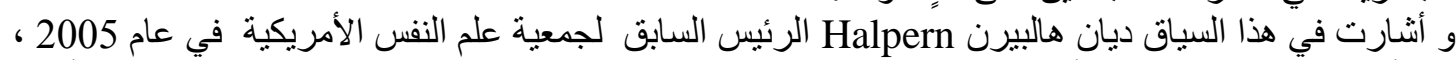

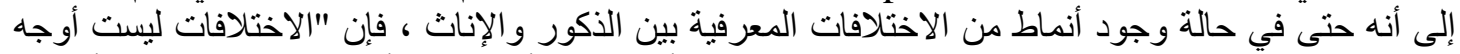

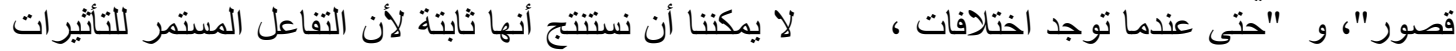

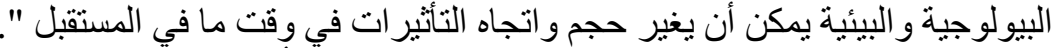

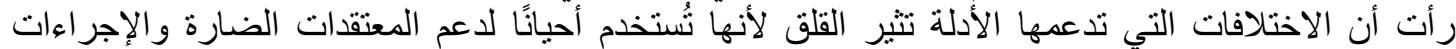

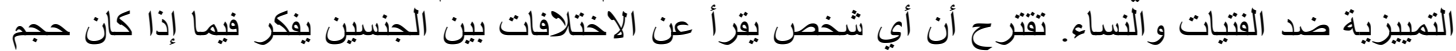

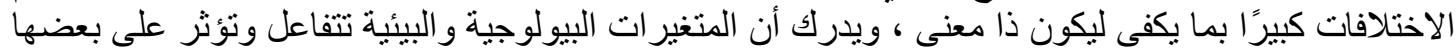

البعض ، و أن الاستتناجات التي نقبلها اليوم يمكن أن تتغير في المستقبل ،

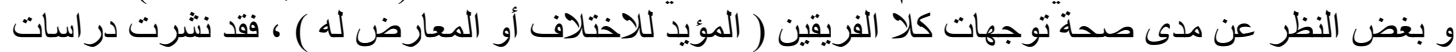

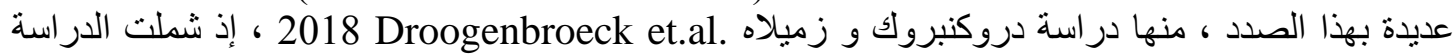

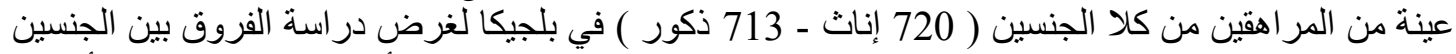

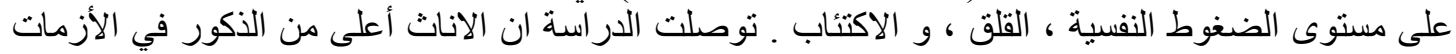

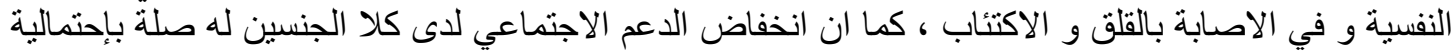

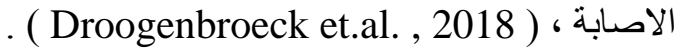
أثر الفروقات الثقافية : اتية

تعد فكرة المرض العقلي و النفسي بحد ذاتها خاضغة للكثير من النقانشات و التمايزات الثقافية بين المجتمعات ،

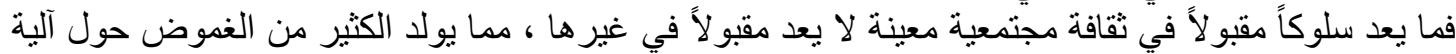
تحديد معايير الصحة العقلية عبر الثقافات المختلفة . فئلة 


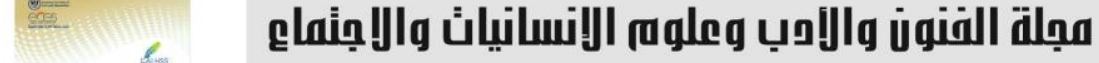 \\ Journal of Arts, Literature, Humanities and Social Sciences www.jalhss.com

و يُسهم التمايز الثقافي عبر أنحاء العالم بالتنأثير في جانب الصحة العقلية للأفراد ، بدءاً بطريقة إدراك أهمية

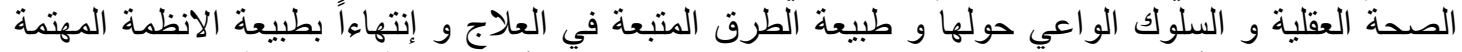

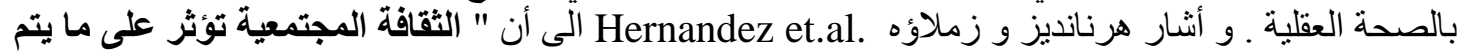

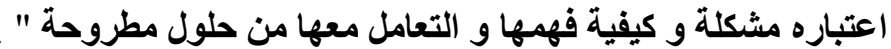

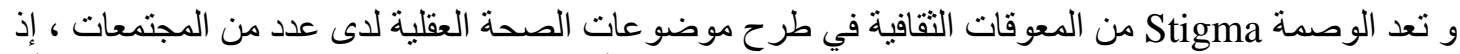

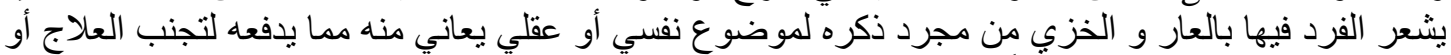

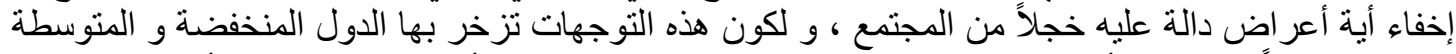

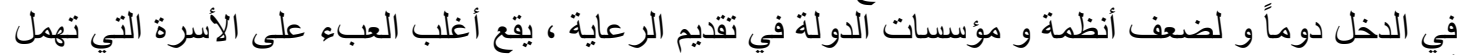

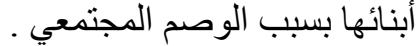

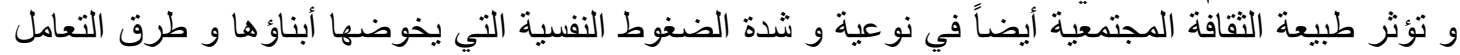

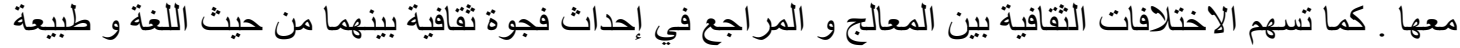

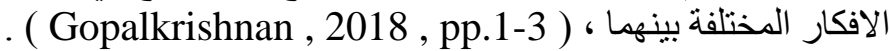

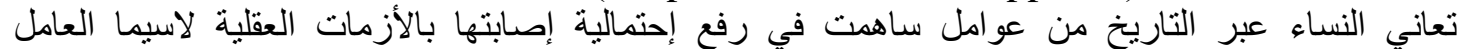

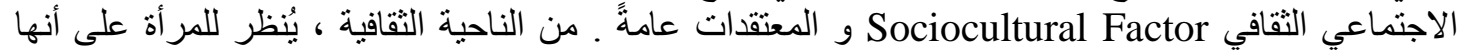

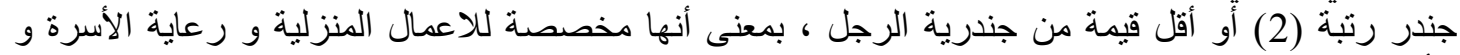

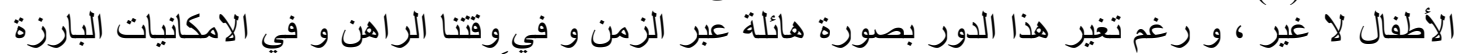

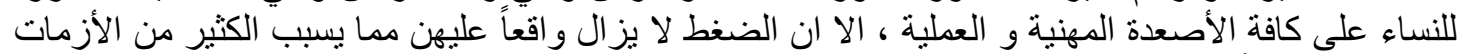

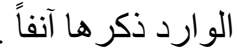

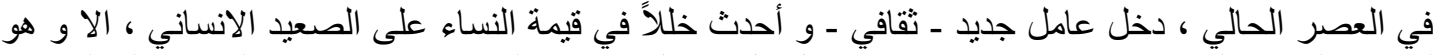

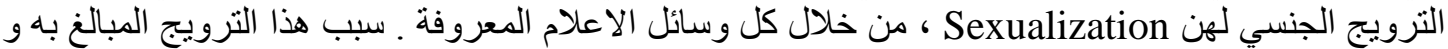

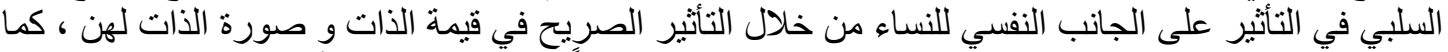
صرحت به الجمعية النفسية الامريكية A.P.A ، و الذي الذي يقود لاحقاً في شعور النساء بأعر اض الاكتئاب و القلق

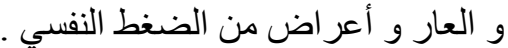

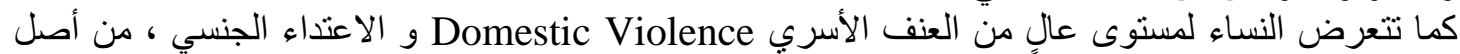

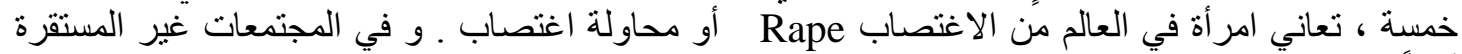

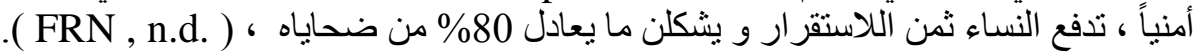

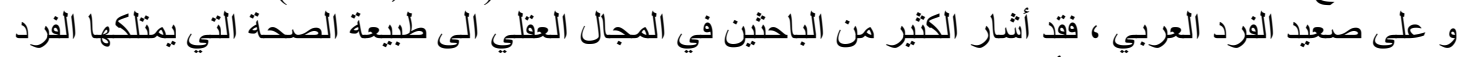

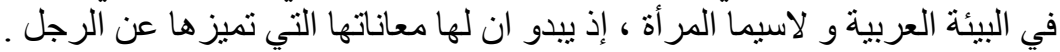

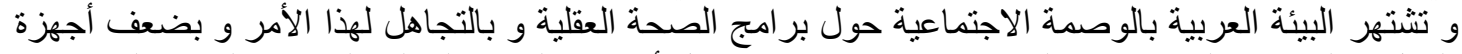

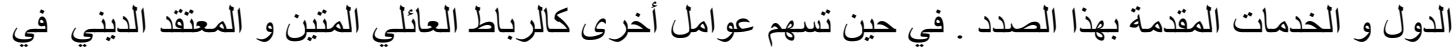
(Yahia , 2012)

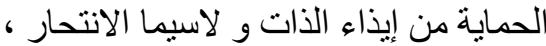

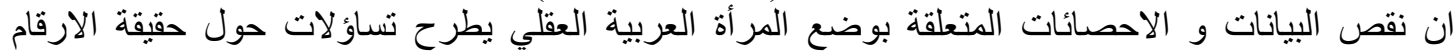

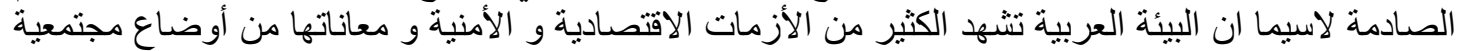

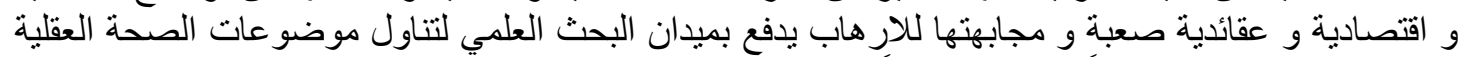

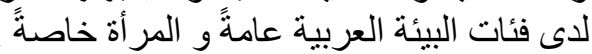

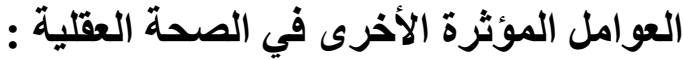

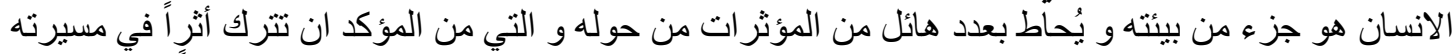

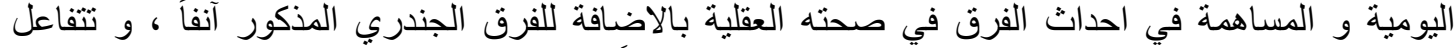

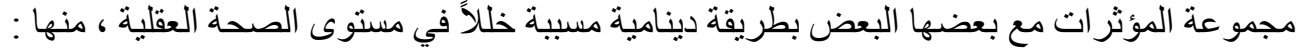

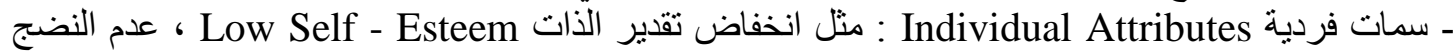

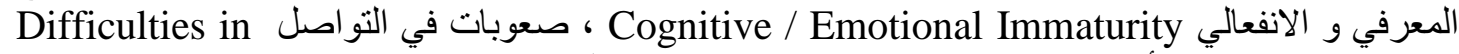

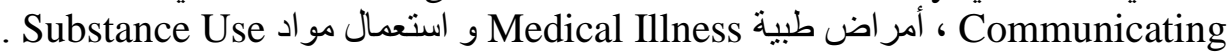

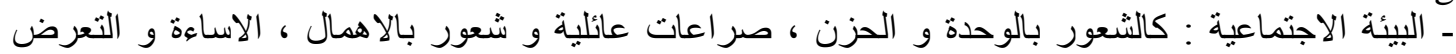

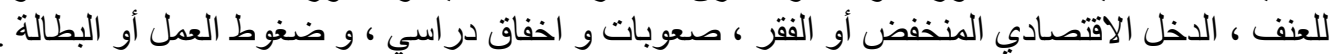




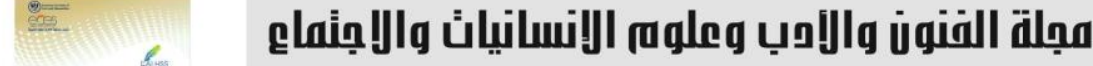 \\ Journal of Arts, Literature, Humanities and Social Sciences www.jalhss.com

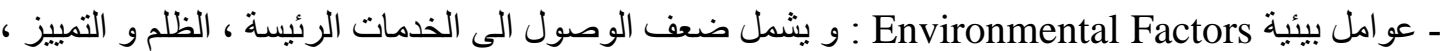

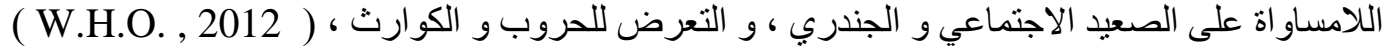

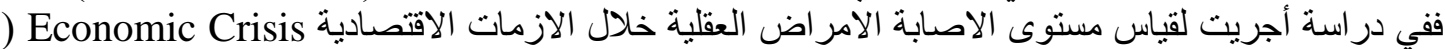

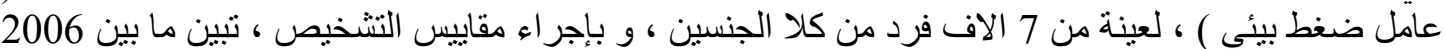

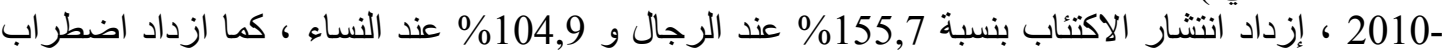

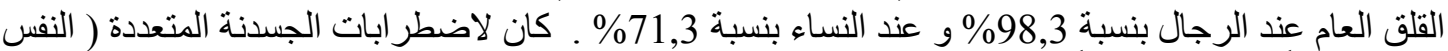

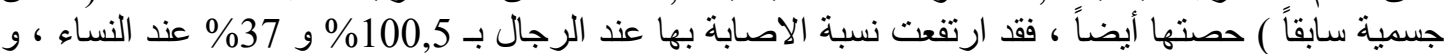

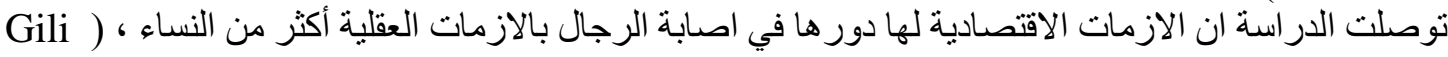
, Lopez- Navaroo , Castro , Homar , Navarro , Garcia - Toro , Garcia - Campayo \& . ( Roca , 2016

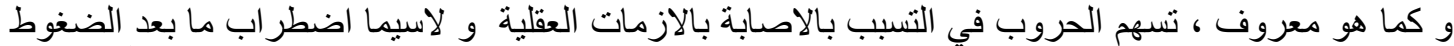

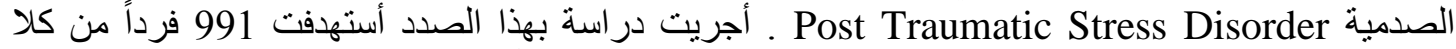

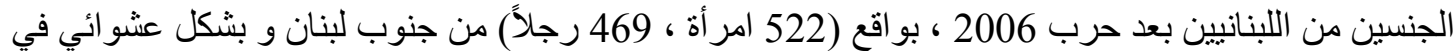

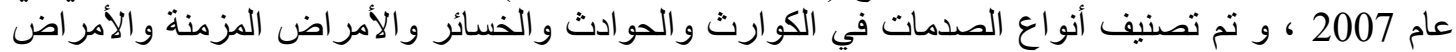

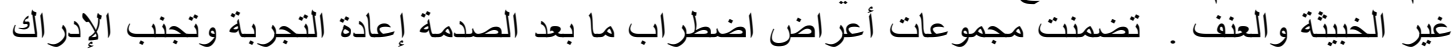

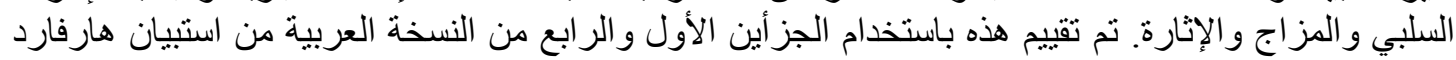

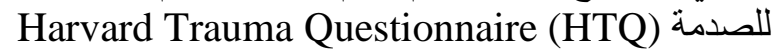
تم تقييم عو امل الخطر باستخدام بيانات من الدعم الاجتماعي واستبيان أحداث الحياة في نماذج الانحدار المتعددة.

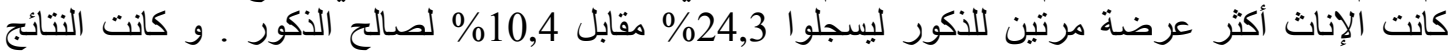

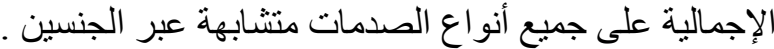

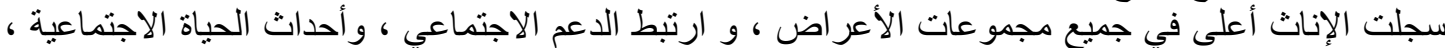

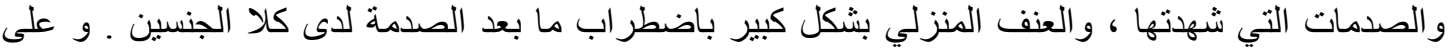

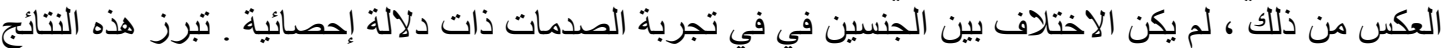

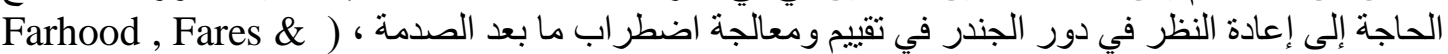
. ( Hamady, 2018

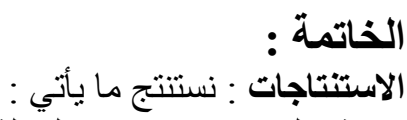

ـ تؤثر الموضو عات ذات الصلة بالصحة العقلية في موارد الدول من حيث توفير مصادر التشخيص و العلاج .

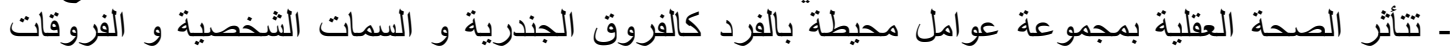

ـ ـ هناك فروق جندرية ـ متضاربة الآر اء حولها ـ فيما يتعلق بنو عية الاضطر ابات العقلية .

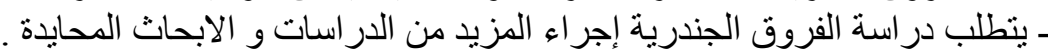

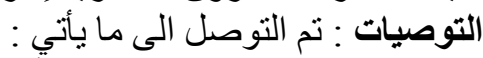

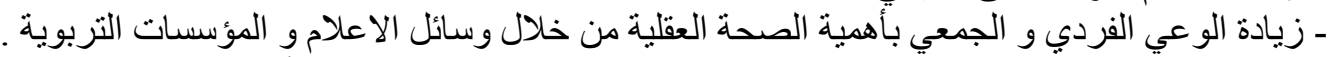
ـ فتح آفاق علمية جديدة للبحث حول طبيعة الفروق بين النساء و الرجال و أسبابها من خلال ميدان البحث العلمي. ـ درأسة طبيعة الظروف الراهنة للبلد و معرفة مدى نأثير ها على سلامة الصحة العقلية لمواطنيه من قبل مؤسسات وزارة الصحة و المؤسسات التعليمية .

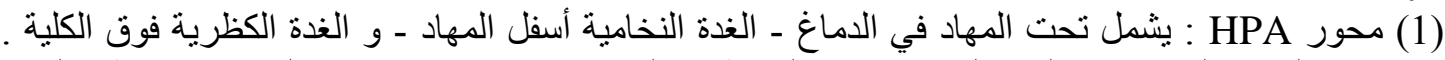

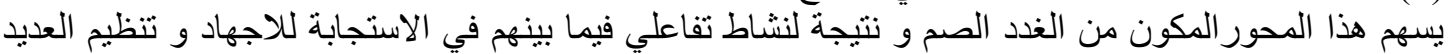

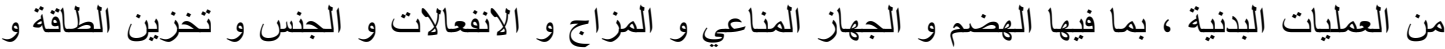




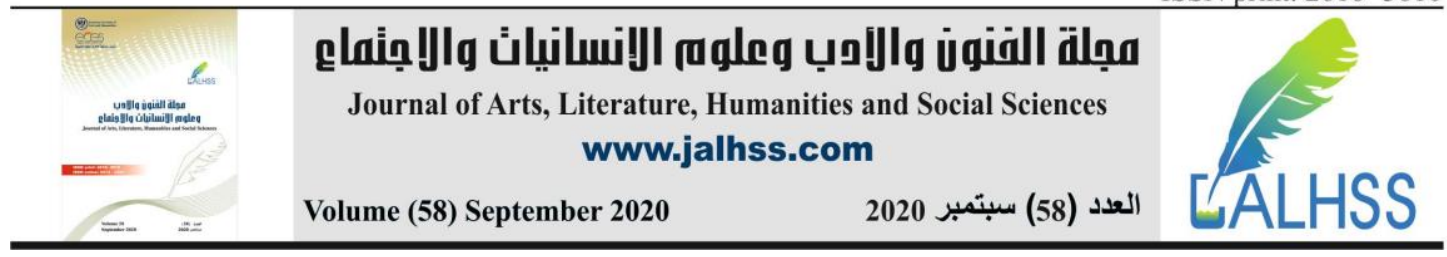

Hans ) : Stress without distress , Philadelphia: Lippincott. ISBN 978-0-397-01026-4 . Seyle $(1974$

\section{References}

1- Altemus , M. ; Sarvaiya , N. \& Epperson, C.N. (2014) : Sex Differences in Anxiety and Depression Clinical Perspectives, Front Neuroendocrinol , Vol.35 , No.3 , pp.1-26.

2- American Psychological Association ( 2005 ) : Men and Women: No Big Difference, https://www.apa.org/research/action/difference .

3- American Psychiatry Association A.P.A.( 2017 ) : Mental Health Facts for Women , apa.org .

4- Barbieri , Norma (1999) : Psychoanalytic Contributions to the study of Gender Issues, Can J. Psychiatry, Vol.44 , No.1 , pp.72-77 .

5- Bertolote, Jose (2008) : The roots of the concept of mental health, World Psychiatry Journal , 7(2) .

6- Britannica (2014) : Mental Hygiene, https://www.britannica.com/science/mentalhygiene

7- Droogenbroeck, Filip Van ; Spruyt, Bram \& Keppens , Gil (2018) : Gender differences in mental health problems among adolescents and the role of social support: results from the Belgian health interview surveys 2008 and 2013 , BMC Psychiatry volume 18, Article number: 6 .

8- Farhood, L. ; Fares , S. \& Hamady , C.(2018) : PTSD and gender : could gender differences in war trauma type, symptom clusters and risk factors predict gender differences in PTSD prevalence? , Archives in Women's Mental Health , Vol.21, No.6 , pp. 725-733 , DOI: 10.1007/s00737-018-0849-7

9- Foundation Recovery Network ( n.d.) : Common Mental Health Issues among Women , https://dualdiagnosis.org/mental-health-and-addiction/common-issueswomen .

10- Gabriel, Phyllis \& Marjo-Riitta Liimatainen ( 2000 ) : Mental Health in the Workplace: Introduction, Executive Summaries, Cornell University ILR School .

11- Gili , Margalida ; Lopez-Navarro , Emilio ; Castro , Adoracion ; Homar, Clara ; Navarro , Capilla ; Garcia - Toro , Mauro ; Garcia - Campayo , Javier \& Roca , Miquel (2016) : Gender differences in mental health during the economic crisis, Psicothema, Vol. 28, No. 4 , pp. 407- 413 , doi: 10.7334/psicothema2015.288

12- Gopalkrishnan , Narayan (2018 ) : Cultural Diversity and Mental Health: Considerations for Policy and Practice, Public Health Journal .

13- Hyde , Janet Shibley (2005) : The Gender Similarities Hypothesis, American Psychologist, The American Psychological Association, Vol.60 , No.6 , pp.581592, OI: 10.1037/0003-066X.60.6.581 .

14- Kamal, Rabah (2017) : What are the current costs and outcomes related to mental health and substance use disorders,

15- Khushalani , Jaya S. (2018) : Systematic review of healthcare costs related to mental health conditions among cancer survivors, Expert Review of Pharmacoeconomics \& Outcomes Research , 18(5) . 


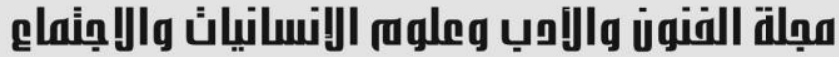 \\ Journal of Arts, Literature, Humanities and Social Sciences www.jalhss.com \\ Volume (58) September 2020 \\ العدد (58) سبتمبر 2020}

16- Lauren , Heidemarie ; Vergara-Lopez, Chrystal \& Stroud, Laura R. (2016) : Differential relations between youth internalizing/externalizing problems and Cortisol responses to performance vs. interpersonal stress, The International Journal on the biology of stress, Vol.19, Issue 5 , https://doi.org/10.1080/10253890.2016.1218843 .

17- Mandell, Wallace (1997) : Origins of Mental Health The Realization of an Idea, Johns Hopkins Bloomberg School of Public Health, jhsph.edu .

18- NIMH (2019) : Women and Mental Health, www.nimh.nih.gov .

19- Northridge , Mary E. (1999) : Mind Matters : The Importance of Mental Disorders in Public Health's 21st Century Mission, American Journal of Public Health, Vol.89, No.9 .

20- Ogrodniczuk, John ; Oliffe , John ; Kuhl , David \& Gross , Paul A. (2016) : Men's Mental Health, Can Fam Physician , Vol.62 , N. 6 , pp. 463-464 , PMCID: PMC4907547, PMID: 27302996.

21- Riecher - Rossler, Anita (2018) : Introduction: Gender-Specific Issues Relative to Mental Illness, Psychiatric Times, Vol.35, Issue 11.

22- Rowan , Karen (2011) : In Mental Illness, Women Internalize and Men Externalize , https://www.livescience.com/15658-mental-illness-women-mendifferences.html .

23- Rosenfield, Sarah \& Smith, Dina (2012) : Gender and Mental Health: Do Men and Women Have Different Amounts or Types of Problems? , Teresa L. Scheid \& Tony N. Brown, A Handbook for the study of Mental Health , Cambridge University Press, 2nd Edition , https://doi.org/10.1017/CBO9780511984945 .

24- Udry, J. Richard (1994) : The Nature of Gender, Demography, Vol. 31 , No.4, pp. 561-573. doi:10.2307/2061790. JSTOR 2061790. PMID 7890091 .

25- World Health Organization W.H.O (2002) : Gender and Mental Health, www.who.int .

26- W.H.O.(2012) : Risks to Mental Health : An overview of Vulnerabilities and Risks Factors, www.who.net .

27- W.H.O (2018) : Mental health: strengthening our response, www.who.int .

28- Yahia , Mohammed (2012) : dealing with Mental Illness in the Middle East, https://www.natureasia.com . 